\title{
The interfacial energy in the Cassie-Baxter regime on the pyramid decorated solid surface
}

\author{
Waldemar Nowicki ${ }^{\mathrm{a}}$ \\ Faculty of Chemistry, Adam Mickiewicz University, Umultowska 89b, 61-714 Poznań, Poland
}

Received 17 January 2019 and Received in final form 18 May 2019

Published online: 4 July 2019

(C) The Author(s) 2019. This article is published with open access at Springerlink.com

\begin{abstract}
The wettability of the solid surface covered with a pattern of pyramids is studied in categories of behavior of a single liquid droplet settled onto such a surface. The Cassie-Baxter regime of wetting is assumed. The cellular model of liquid-solid interface has been proposed. On the basis of the calculations for a single elemental cell with applied periodic boundary conditions, the components of the interfacial energy have been obtained. The dependence of the interfacial energy and hypothetical pseudo-contact angle on the hydrostatic pressure is discussed. The influence of the line tension on the wettability of the surface, expressed in terms of the apparent contact angle is shown.
\end{abstract}

\section{Introduction}

The hydrophobic surfaces decorated with micro- and nanoscale roughness mimicking the non-adhesive plant leaves [1] show useful and unique properties [2]. These properties have attracted a lot of interest due to their potential application in self-cleaning [3], anti-icing [4], antifouling [5], drag reduction [6], membranes for water-oil separation [7] to name a few. The properties follow from the specific way of wetting the surfaces - the liquid wets only the upper parts of the surface irregularities and does not fill the void between them, leaving an air layer underneath [6]. The reduced contact area between the liquid and the surface results in unusual behavior of a water droplet settled on the surface such as large contact angles and negligible contact angle hysteresis [6].

The physical characteristics of wetting properties are usually based on parameters well defined for smooth surfaces such as the contact angle, the surface or interface tension, the free surface energy, the work of adhesion etc. However, some of them seem to be ill defined in the case of rough surfaces. It seems that the measurable mean values of these parameters do not give an insight into details of wetting in the microscale, where the surface phenomena are influenced by gravity, even in the case of droplets of the size from the capillary regime (i.e., at small Bond number). The simulations presented in this work are an attempt at getting insight into the actual values of the parameters hidden from direct measurements.

The thermodynamic equilibrium contact angle on an ideal smooth and energetically homogenous solid surface

\footnotetext{
a e-mail: gwnow@amu.edu.pl
}

is defined by the Young equation [8]

$$
\cos \left(\theta_{\mathrm{Y}}\right)=\frac{\gamma_{\mathrm{SV}}-\gamma_{\mathrm{SL}}}{\gamma_{\mathrm{LV}}},
$$

where $\gamma$ is the interfacial tension and indices SV, SL and LV relate to the solid-vapor, solid-liquid and liquid-vapor interfaces, respectively.

The morphology and energetics of a droplet settled onto a rough surface are influenced by the details of the roughness geometry. When the liquid penetrates the whole surface under the droplet, the wetting occurs in the socalled Wenzel regime [9] In such a case, when the surface irregularities are small as compared to the size of the droplet, the apparent contact angle is defined by the Wenzel law [10]:

$$
\cos \left(\theta_{\mathrm{W}}\right)=r \cos \left(\theta_{\mathrm{Y}}\right),
$$

where $r$ is the ratio of the rough surface area to the area of the surface projection on the parallel plane.

When the surface under the droplet is not fully wetted, the wetting is known as occurring in the Cassie-Baxter regime [11]. The apparent contact angle in the Wenzel and Cassie-Baxter regimes takes the general form [12]:

$$
\cos \left(\theta_{\mathrm{WCB}}\right)=r f \cos \left(\theta_{\mathrm{Y}}\right)-(1-f),
$$

where $f$ stands for the fraction of the liquid/solid interface area and $1-f$ is the fraction of the liquid/vapor interface area. At $r=1$, eq. (3) is equivalent to the Cassie law $[13,14]$.

There are a lot of regular patterns (for example surfaces decorated with rectangular bars shown in fig. 1(a)) on which the droplet behaves exactly in the way determined by eq. (3) at $r=1$. The stability of a droplet 
a)

\section{MMNMMUMA}

Fig. 1. The morphology of the droplet settled on the surface decorated by cuboid pillars (a) and pyramids or cones (b) in the Cassie-Baxter regime. The latter pattern does not contain edges at which the pining effect may take place and the liquid can slip down on solid faces. a)

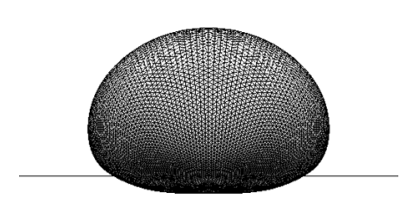

b)

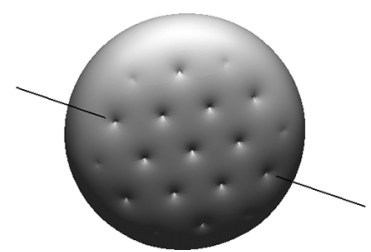

b)

Fig. 2. Morphology of a droplet of volume $V=50 \mathrm{~mm}^{3}$ on the surface decorated with cones in the Wenzel-Cassie-Baxter regime: projection of the droplet on the vertical plane (a) and view of the droplet bottom (b). The solid surface is not shown. Instead the line drawn at the elevation of cone tops is visible. Parameters of simulation: $\gamma_{\mathrm{LV}}=72.4 \cdot 10^{-3} \mathrm{~N} / \mathrm{m}$, density $\rho=$ $10^{-3} \mathrm{~kg} / \mathrm{m}^{3}$, acceleration due to gravity $g=9.81 \mathrm{~m} / \mathrm{s}^{2}$.

deposited onto the surface is mainly caused by the pinning $[15,16]$ of the liquid surface on the sharp edges of cuboids. However, the system may not be in the thermodynamic equilibrium - it can be switched to the Wenzel regime, when the pinning is broken and the liquid fills the grooves between cuboid pillars $[10,17]$.

In systems in which the position of the liquid/vapor interface is not stabilized by the pinning effect (for an example see fig. 1(b)) or the pinning can be easily broken, the $r$ parameter in eq. (2) is greater than 1 . The $f$ parameter may be influenced by the pressure exerted on the liquid surface at the droplet basis. This pressure, being the superposition of the hydrostatic and the Laplace pressures, pushes down the liquid surface and causes wetting of parts of solid surface. As follows from the Pascal law, the hydrostatic pressure on the whole droplet basis is the same and determined by the elevation of the top of the droplet. However, the mean curvature of the bottom of the droplet surface may change smoothly as a result of the Laplace pressure.

The morphologies of the water droplets deposited on the solid surface decorated with cones in the WenzelCassie-Baxter regime are shown in figs. 2-4. The morphologies are obtained from simulations performed for the system of 19 cones located at distances of $1 \mathrm{~mm}$ in the hexagonal lattice by the SE (Surface Evolver $[18,19]$ ) software. The slope of cone surface was $0.611 \pi\left(110^{\circ}\right)$, whereas the Young contact angle on the cone surface was $\theta_{\mathrm{Y}}=2 / 3 \pi\left(120^{\circ}\right)$. Three different cases were considered: the morphologies of droplets of volumes $V$ equal to 50 and $100 \mathrm{~mm}^{3}$ deposited on the surface without ad- a)

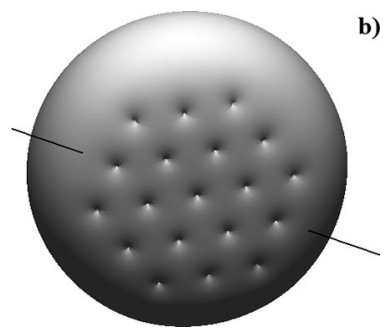

Fig. 3. Morphology of a droplet of volume $V=100 \mathrm{~mm}^{3}$ on the surface decorated with cones in the Wenzel-Cassie-Baxter regime: projection of the droplet on the vertical plane (a) and view the droplet bottom (b). Other simulation parameters are the same as in fig. 2 .

ditional constraints and the morphology of the droplet of $V=300 \mathrm{~mm}^{3}$ located at the same surface but inside the vertical cylinder of radius $0.003 \mathrm{~mm}$ and $\theta_{\mathrm{Y}}=\pi$.

It is evidenced that the cones are dipped in the liquid to different levels depending on their positions (see fig. 2) and the droplet volume (compare figs. 2 and 3 ). The bottom of the droplet of $50 \mathrm{~mm}^{3}$ includes some deep dents caused by cone peaks at the central part of the bottom, whereas peripheral cones are less immersed in the liquid. The immersion of cones in the droplet of $V=100 \mathrm{~mm}^{3}$ is more uniform. However, the cones located far from the droplet center are still less dipped. Although the en$\mathrm{ergy}^{1}$ of solid/liquid/vapor interface under the droplet is high and similar to $\gamma_{\mathrm{LV}}\left(0.077\right.$ and $\left.0.076 \mathrm{~J} / \mathrm{m}^{2}\right)$, the apparent contact angles ${ }^{2}$ are equal to $162^{\circ}$ and $151^{\circ}$ for $V=50 \mathrm{~mm}^{3}$ and $100 \mathrm{~mm}^{3}$, respectively. The maximum immersion depth $h$ (see footnote ${ }^{3}$ ) of the cones into the liquid phase was equal to 0.35 and $0.36 \mathrm{~mm}$, respectively.

When the droplet of larger volume $\left(V=300 \mathrm{~mm}^{3}\right)$ is located inside the cylinder, which makes it impossible to spread over the larger area of the surface, the hydrostatic pressure at the droplet bottom is higher and the immersion depth increases evidently to $h=0.66 \mathrm{~mm}$ (see fig. 4). However, the apparent contact angle observed is still small and even decreases to the value of $131^{\circ}$ as a result of boundary conditions introduced by cylinder walls. The interfacial energy at the droplet bottom is relatively high and equal to $0.089 \mathrm{~J} / \mathrm{m}^{2}$.

The above results suggest that the apparent contact angle is not a credible measure of the solid/liquid/vapor interface energy at the droplet.

The exact simulation of the behavior of the systems depicted in figs. 2-4 is challenging, since the question whether the liquid surface would wet the specified cone or not is difficult to answer, especially under the periph-

1 The interfacial energy is assumed to be equal to the energy of the part of the droplet surface located below the cone peaks normalized to $1 \mathrm{~m}^{2}$.

2 The contact angle is assumed to be the angle between tangent to liquid surface at the level of cone peaks (given by slopes of appropriate lattice triangles representing the free liquid surface) and the horizontal line passing through the peaks.

3 The immersion depth is the difference in the elevation on the cone peaks and the lowest point of the liquid surface. 


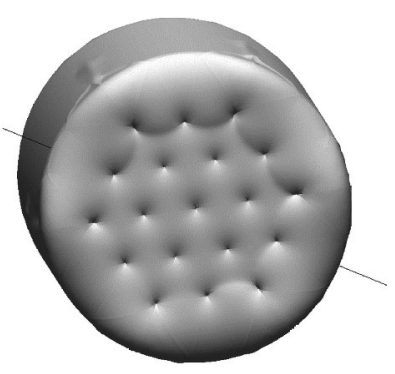

Fig. 4. Morphology of a droplet of volume $V=300 \mathrm{~mm}^{3}$ inside a cylinder located on the surface decorated with cones in the Wenzel-Cassie-Baxter regime. The view shows the droplet bottom. Other simulation parameters are the same as in fig. 2.

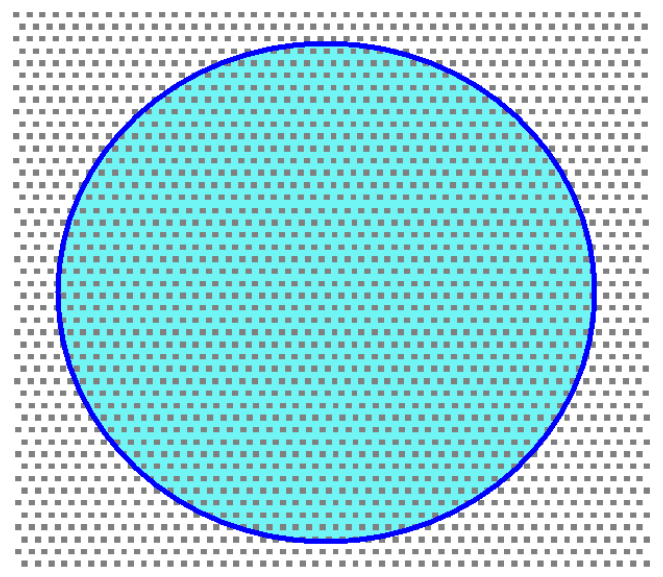

Fig. 5. Schematic view of the droplet deposited onto a pyramid decorated solid surface.

eral parts of the droplet bottom. The computation needs many trials performed at different constraints imposed on the liquid surface. Instead, the simulation of wetting phenomena occurring at the central part of the droplet basis (where the immersion of cones does not depend on their position) is easier to perform.

In the present study an attempt was made to verify the hypothesis that the wetting phenomena under the droplet settled onto the solid surface decorated with pyramids of the size much smaller than the size of the droplet, forming a quasi-hexagonal mosaic (see fig. 5) are remarkably influenced by the hydrostatic pressure $p_{\mathrm{hs}}$ of the liquid and by the line tension $\sigma_{\mathrm{SLV}}$. In the studied system, there are no pinning edges impeding the vertical movement of the liquid surface on the surface irregularities. The aim of the study is determination of the relations between $p_{\mathrm{hs}}$ and $\sigma_{\mathrm{SLV}}$, and the morphology and energetics of the liquid/solid/vapor interface [20]. The study was performed by means of simulations with the finite element method, using the Surface Evolver (SE) software [18, 19], by minimization of free interfacial energy of the liquid/solid/vapor interfaces at the bottom of the droplet. Since the numerical calculation for the whole system - the droplet and the solid surface covered with tens or hundreds pyramids, as shown in fig. 5, is extremely difficult, all calculations were performed for a periodic elemental cell

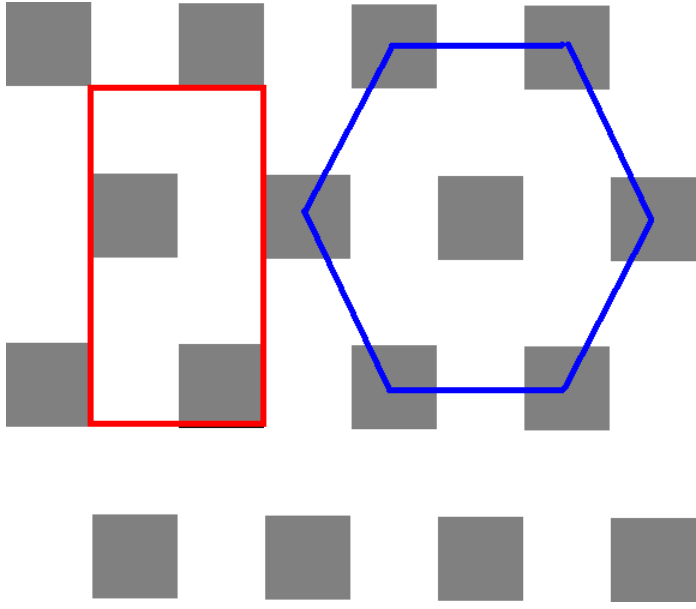

Fig. 6. The scheme of quasi-hexagonal surface coverage with pyramids. The red line denotes the elementary periodic cell of the coverage.

of the surface containing only two pyramids. The chosen method gives the precise values of parameters describing the wettability phenomena at the droplet bottom except for the neighborhood of the bottom perimeter.

The fragment of the surface with the unit cell marked with the red line is presented in fig. 6 .

For simplicity, the pure Wenzel regime of wetting was neglected in the computation. The extension of the wetting range to this region would require additional constrains and would greatly reduce the convergence of calculations. In addition, results from this range are nor relevant to the studied problem.

The paper is organized as follows: firstly, the basic assumptions of the SE model applied to the simulation of the liquid/vapor and liquid/solid interfaces behavior are introduced (sect. 2). Then, in sects. 3.1 and 3.2 the obtained relationships between applied pressures and changes induced by them in the system morphology and energetics are discussed. The effect of the line tension on the wettability is shown in sect. 3.3. Finally, the conclusions drawn from the work are presented in sect. 4 .

\section{Model}

The simulation box is an elementary cell shown in details in fig. 7 . The cell contains two pyramids located at $x=2.5 \cdot 10^{-6} \mathrm{~m}, y=2.5 \cdot 10^{-6} \mathrm{~m}$ and $x=7.5 \cdot 10^{-6} \mathrm{~m}, y=$ $17.5 \cdot 10^{-6} \mathrm{~m}$ on a rectangle of the size $1 \cdot 10^{-5} \mathrm{~m} \times 2 \cdot 10^{-5} \mathrm{~m}$. The slope angle $\alpha$ of all pyramid faces is $0.611 \pi\left(110^{\circ}\right)$. The upper part of the cell is filled with water to the level forming the liquid/vapor interface. The liquid/vapor interfacial tension is assumed as equal to $\gamma_{\mathrm{LV}}=72.4 \cdot 10^{-3} \mathrm{~N} / \mathrm{m}$, density $\rho=10^{-3} \mathrm{~kg} / \mathrm{m}^{3}$. The Young contact angle on the pyramid faces is $\theta_{\mathrm{Y}}=2 / 3 \pi\left(120^{\circ}\right)$. Periodic boundaries at all vertical walls of the simulation box are assumed (the torus model $[18,19])$. The bottom of the simulation box was not incorporated into the system, subsequently the computation neglects the Wenzel regime of wetting. 


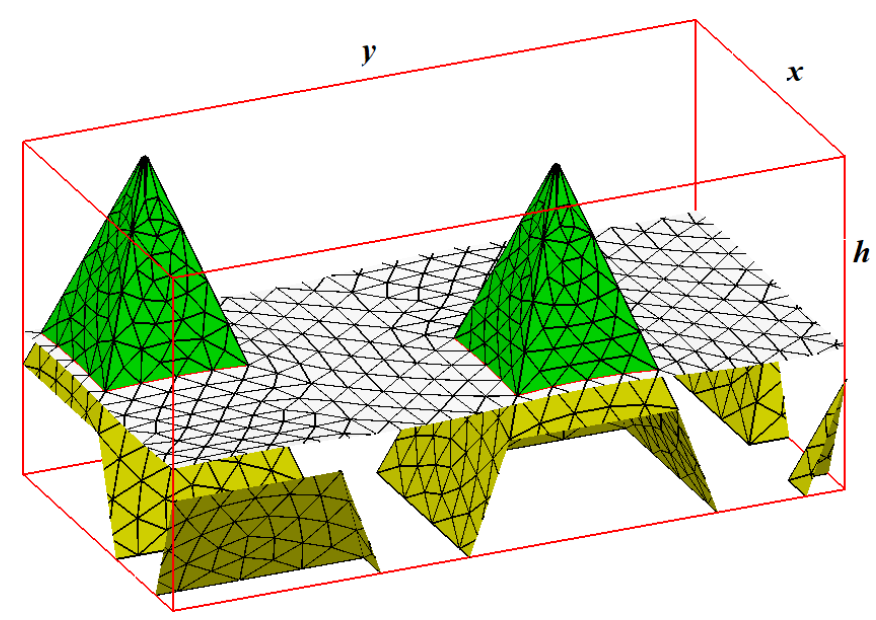

Fig. 7. The view of a single elementary cell in the course of the simulation $\left(\sigma_{\mathrm{SLV}}=0 \mathrm{~N}, p=9.81 \mathrm{~N} / \mathrm{m}^{2}\right)$.

In the section devoted to the influence of the line energy on the behavior of droplet on pyramids, the positive line tension from the range $\sigma_{\mathrm{SLV}}=0-2 \cdot 10^{-8} \mathrm{~N}$ is applied [21].

The elements of the surface components are represented by a mesh of triangles. The SE program minimizes the free energy of the modelled system in defined steps including procedures of mesh refinement, vertex averaging, polishing up the triangulation and the energy minimization by means of the conjugated gradient descent method and the Newton method $[18,19]$. Mechanically stable interface configurations are obtained by minimizing the sum of all interfacial energies, which is a function of the coordinates of the nodes.

Minimization steps include a sequence of conjugated descents and hessian_seek commands $[18,19]$. At the end of each simulation, a couple of Hessian commands (switching on the Newton method of the free energy minimization) are executed. The final morphology of all interfaces in the simulation box consists of roughly 3000 individual nodes.

When the interfaces reached their final equilibrium morphology, the number of selected quantities was calculated by means of internal variables and attributes of geometric elements of SE. These quantities include: geometrical dimensions of the interfaces and their free energies.

The total free energy of the solid/liquid/vapor interface under the droplet is calculated as the sum of the products of interface area and interface tension for each element of the lattice. The total free interface energy $E_{\mathrm{T}}$ of the fragment of the droplet bottom located in the simulation box can then be expressed as

$$
E_{\mathrm{T}}=E_{\mathrm{LV}}+E_{\mathrm{SL}}=\gamma_{\mathrm{LV}}\left(A_{\mathrm{LV}}+\cos \left(\theta_{\mathrm{Y}}\right) A_{\mathrm{SL}}\right)
$$

where $E_{\mathrm{LV}}$ and $E_{\mathrm{SL}}$ are the free energies of the liquid/vapor and solid/liquid interfaces at the droplet bottom, $A_{\mathrm{LV}}$ and $A_{\mathrm{SL}}$ are the areas of these interfaces, respectively.

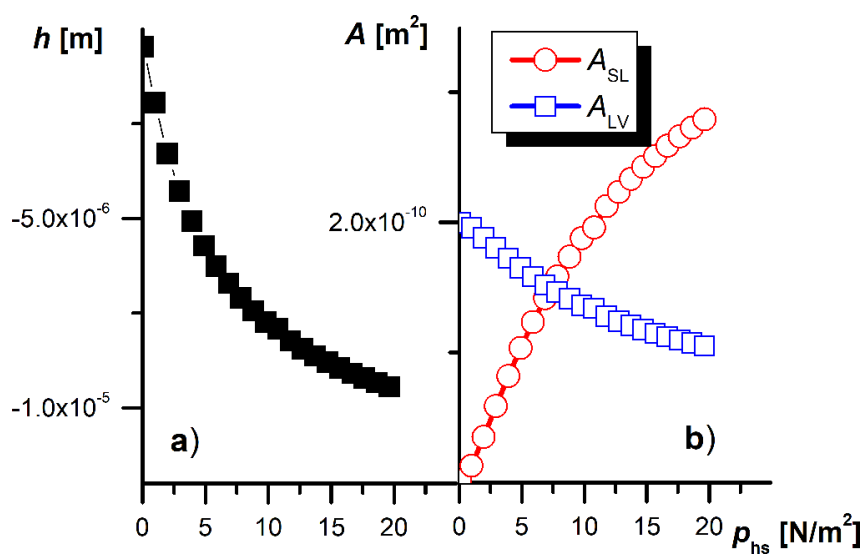

Fig. 8. The dependence of the liquid/vapor elevation (a) and the liquid/solid and liquid/vapor interface areas (b) on the hydrostatic pressure $\left(\sigma_{\mathrm{SLV}}=0 \mathrm{~N}\right)$.

\section{Results}

\subsection{Morphology of cell components}

In the computation of the interface morphology and energetics, the reference system was assumed, in which the hydrostatic pressure $p_{\mathrm{hs}}$ in the liquid at the level corresponding to the tops of the pyramids (assumed as $h=0$ ) is equal to zero. In such a case, since the surface is hydrophobic $\left(\theta_{\mathrm{Y}}=2 / 3 \pi\right)$, the liquid/solid interface is flat and touches pyramids only at single points. When hydrostatic pressure increases, the elevation of the interface decreases and, at the same time, the average convexity of the free liquid surface increases. The lowest point of the hanging interface is located at a halfway between the pyramids as shown in fig. 7 .

The dependence of the elevation $h$ of this point on the hydrostatic pressure shown in fig. 8(a) is, as expected, a monotonically decreasing function. The areas of both interfaces of interest are shown in fig. 8(b). With increas$\operatorname{ing} p_{\mathrm{hs}}$, the solid/liquid interface area increases from zero, whereas the liquid/vapor interface area decreases. The last part of the dependences is not included since it would concern the transition to the neglected Wenzel regime.

\subsection{Energetics of interfaces}

Figure 9(a) presents the free energies of the interfaces of interest included in the simulation box. Both components of the total free energy of the system - the energies of the liquid/vapor and liquid/solid interfaces behave similarly to the interface areas depicted in fig. 8(b). The $E_{\mathrm{T}}$ value monotonically increases as a result of increasing "roughness" of the liquid surface caused by the solid pyramids sticking into it. This increase is a result of the work $W$ done by the hydrostatic pressure, consisting in lowering the height of the liquid surface by $h$ (equivalent to the 


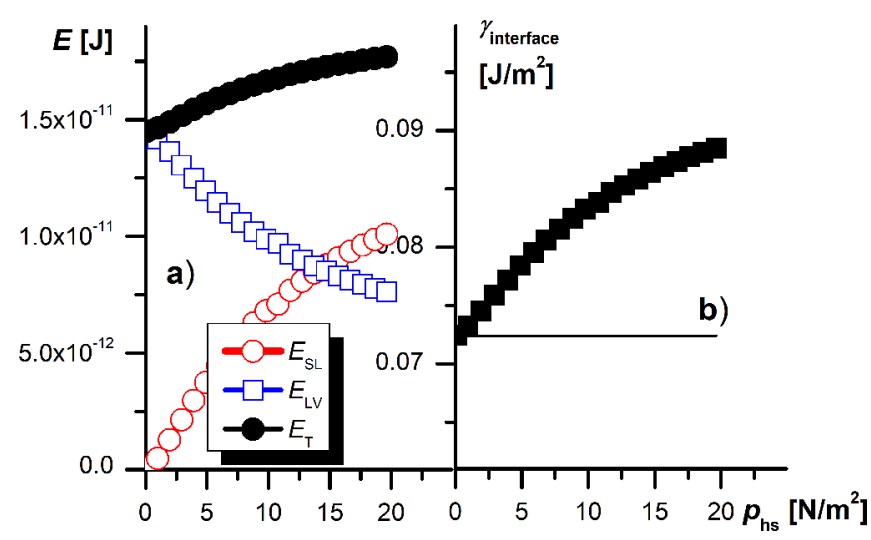

Fig. 9. The dependence of the total interfacial energy and its components: the liquid/solid and liquid/vapor interface energies (a) and the average interfacial tension (b) on the hydrostatic pressure $\left(\sigma_{\mathrm{SLV}}=0 \mathrm{~N}\right)$.

immersion depth of pyramids into liquid):

$$
W=-p_{\mathrm{hs}} V=-\rho g h V=-\rho g\left(A_{\mathrm{P}}-\left(\frac{2 h}{\tan (\pi-\alpha)}\right)^{2}\right) h^{2},
$$

where $A_{\mathrm{P}}=x y$ is the surface area of the projection of the interface onto the horizontal plane (the area of the bottom of elementary cell) shown in fig. 7, $V$ is the volume of displaced liquid. Namely, $V$ is the volume of the $x y h$ cuboid (see fig. 7) reduced by the volume of the wetted parts of pyramids (marked in green). Equation (5) is only an approximation of $W$ but a fairly good one considering that the free liquid surface is almost flat (at $\alpha \approx \theta_{\mathrm{Y}}$ ) as shown in fig. 7 .

The average interfacial tension in the elementary cell (fig. 9(b)) equivalent to the average interfacial tension at the bottom of the droplet was calculated from the equation

$$
\begin{aligned}
\gamma_{\text {interface }} & =\frac{\gamma_{\mathrm{LV}} A_{\mathrm{LV}}+\left(\gamma_{\mathrm{LS}}-\gamma_{\mathrm{SV}}\right) A_{\mathrm{SL}}}{A_{\mathrm{P}}} \\
& =\frac{\gamma_{\mathrm{LV}}\left(A_{\mathrm{LV}}-\cos \left(\theta_{\mathrm{Y}}\right) A_{\mathrm{SL}}\right)}{A_{\mathrm{P}}}
\end{aligned}
$$

The interfacial tension increases with increasing immersion depth producing the repulsion force exerted by the solid surface decorated with pyramids to the liquid surface. The force compensates the weight of the liquid and can be related to the repulsion pressure $p_{\text {rep }}$ equal to the partial derivative of the mean interfacial tension over the immersion depth:

$$
p_{\text {rep }}=\left(\frac{\partial \gamma_{\text {interface }}}{\partial h}\right)_{T, V}
$$

The higher the hydrostatic tension, the higher the average interfacial energy. The solid surface hydrophobicity increases, which can be illustrated by the increase in the hypothetical contact angle $\theta_{\text {ph }}$ which might be observed if the triple line were laying on the bottom of cylinder in which the droplet is located. This hypothetical pseudo-contact angle, which can be interpreted as additional thermodynamic parameter characterizing the solid surface hydrophobicity, could be calculated assuming different surface tensions of the "phantom" SV interface. Hence, assuming that the "phantom" interface tension is equal to zero

$$
\cos \left(\theta_{\mathrm{ph}}\right)=\frac{0-\gamma_{\text {interface }}}{\gamma_{\mathrm{LV}}}
$$

$\theta_{\mathrm{ph}}$ takes complex values almost in the whole range of the hydrostatic pressure studied by the simulations, since, except for very small $p_{\mathrm{hs}}, \cos \left(\theta_{\mathrm{ph}}\right)<-1$. A similar result can be obtained analytically from eq. (3). For the roughness parameter of the surface decorated with pyramids equal to

$$
r=\frac{1}{\cos (\pi-\alpha)}
$$

and the liquid/solid interface fraction expressed as

$$
f=\frac{8 h^{2} \tan \left(\alpha-\frac{\pi}{2}\right)}{x y \cos \left(\alpha-\frac{\pi}{2}\right)}
$$

(which is a flat liquid surface approximation valid at $\left.\alpha \approx \theta_{\mathrm{Y}}\right)$ the $\theta_{\mathrm{WCB}}=f(h)$ is a straight line dependence starting from the value of $\pi$ and tending to $\theta_{\max }=$ $a \cos \left(r \cos \left(\theta_{\mathrm{Y}}\right)\right)=\pi-0.928 \mathrm{i}$ which relates to the Wenzel regime (eq. (2)).

The hypothetical pseudo-contact angles mentioned above refer to the contact angle observed when the surface texture is impregnated with liquid as a result of the hydrostatic pressure [22]. The imaginary part of the angle is a measure of the force repelling the droplet from the solid surface [23]. However, the pseudo-contact angles with the imaginary parts have no physical meaning in the studied system. When, instead of eq. (8), one assumes the "phantom" tension as equal to $\gamma_{\mathrm{LV}}$, the pseudo-contact angle $\theta_{\mathrm{ph}}$ defined by

$$
\cos \left(\theta_{\mathrm{ph}}\right)=\frac{\gamma_{\mathrm{LV}}-\gamma_{\text {interface }}}{\gamma_{\mathrm{LV}}}
$$

takes only real values.

Figure 10 shows that the pseudo-contact angle vs. hydrostatic pressure dependence is an increasing function and the results obtained in the studied range of $p_{\mathrm{hs}}$ vary approximately from $90^{\circ}$ to $104^{\circ}$. This indicates that the studied solid surface is more hydrophobic than the liquid/vapor interface in the Cassie-Baxter regime.

\subsection{Line tension}

It is widely considered that the effect of the line tension can be observed only for very small droplets located on smooth surfaces when the line energy given by the product of length of the droplet perimeter (the length of the triple line) and the effects of line tension are comparable to those of the interfacial tension. However, in the system 


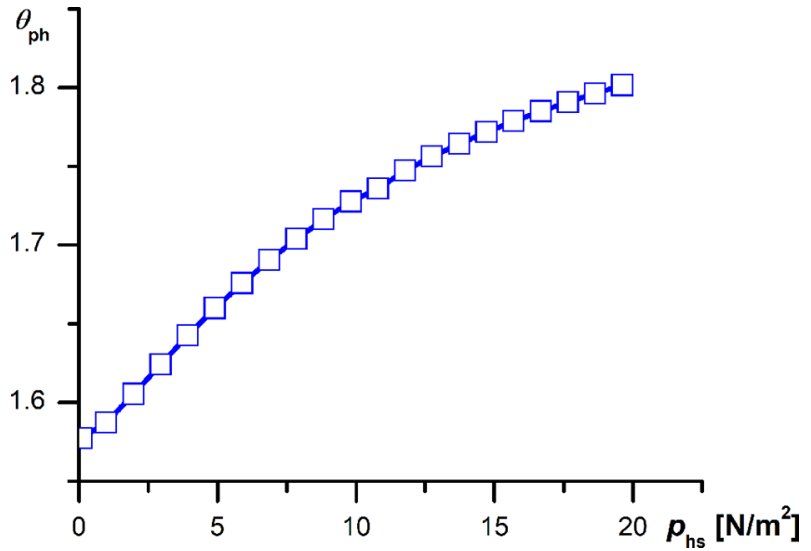

Fig. 10. The dependence of the pseudo-contact angle (eq. (11)) on the hydrostatic pressure $\left(\sigma_{\mathrm{SLV}}=0 \mathrm{~N}\right)$.

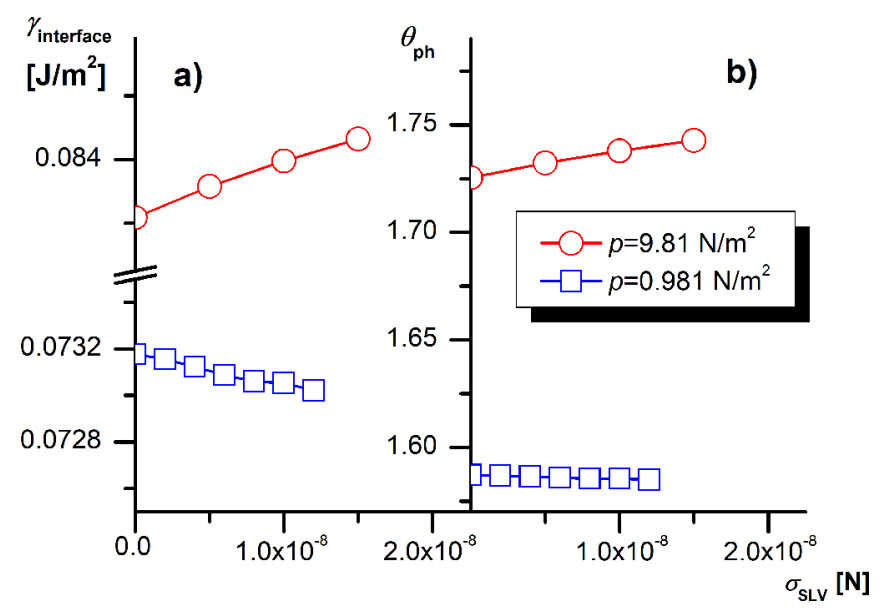

Fig. 11. The dependence of the average interfacial tension (a) and the pseudo-contact angle (b) on the line tension.

considered here, the triple line length is much longer than the droplet perimeter and it is equal to the sum of triple line sections around all pyramids.

Figure 11 presents the results of calculations performed assuming the constant hydrostatic pressure $p$ and constant $\gamma_{\mathrm{SV}}{ }^{-} \gamma_{\mathrm{LV}}$ defined by $\theta_{\mathrm{Y}}$ in the form of the dependence of $\gamma_{\text {interface }}=f\left(\sigma_{\mathrm{SLV}}\right)$ (fig. $\left.11(\mathrm{a})\right)$ and $\theta_{\mathrm{ph}}=f\left(\sigma_{\mathrm{SLV}}\right)$ (fig. 11(b)). Both variables were calculated similarly to the way presented above but taking into account the line energy:

$$
\gamma_{\text {interface }}=\frac{\gamma_{\mathrm{LV}}\left(A_{\mathrm{LV}}-\cos \left(\theta_{\mathrm{Y}}\right) A_{\mathrm{SL}}\right)+\sigma L}{A_{\mathrm{P}}},
$$

where $L$ is the total triple line length in the elemental cell.

According to eq. (12), as a result of the introduction of the non-zero line tension, the increase in the average interfacial tension should be expected since the line energy brings a positive contribution to $\gamma_{\text {interface }}$. Indeed, as shown in fig. 11(a), the increase in $\sigma_{\mathrm{SLV}}$ induces the growth of $\gamma_{\text {interface }}$ when the hydrostatic pressure is relatively high. However, at low $p$, the line tension tending to shorten the length of the triple line raises the level of the liquid surface and, consequently, reduces the solid/liquid interface area and the mean interfacial tension. As shown in fig. $11(\mathrm{a}), \gamma_{\text {interface }}=f\left(\sigma_{\mathrm{SLV}}\right)$ at low $p$ is a descending function. Accordingly, the increase in line tension causes changes in the hydrophobicity of the surface, as illustrated by the pseudo-contact angles (fig. 11(b)).

The results show that the line tension effect (at $\sigma_{\mathrm{SLV}}=$ $2 \cdot 10^{-8} \mathrm{~N}$ ) on the surface decorated by micrometer-scale pyramids may affect the wettability of the surface and can change the values of the pseudo-contact angle by up to $0.05 \pi\left(\sim 1^{\circ}\right)$.

\section{Conclusions}

The thermodynamic parameters characterizing the interfacial properties of the system of the liquid droplet settled on the solid surface decorated with pyramids depend on the hydrostatic pressure exerted onto the droplet bottom. For regular rough surfaces for which the constraints introducing the pinning effect do not apply, but the wetting takes place in the Cassie-Baxter regime (e.g., for the droplet settled on the solid surface decorated with pyramids), the obtained average interfacial tension under the droplet can be much higher than that of the liquid surface tension.

The simulation results shows that the wettability characterized by the mean interfacial energy at the bottom of the droplet is not only related to the roughness, chemical homogeneity and other surface properties, but it is also a function of the hydrostatic pressure exerted by the droplet on the solid surface.

The model presented gives the possibility to incorporate, as an independent parameter, the line tension to the system: liquid-solid surface. The computation shows a small but significant influence of line tension on the average interfacial tension and pseudo-contact angle even for relatively large droplets.

Publisher's Note The EPJ Publishers remain neutral with regard to jurisdictional claims in published maps and institutional affiliations.

Open Access This is an open access article distributed under the terms of the Creative Commons Attribution License (http://creativecommons.org/licenses/by/4.0), which permits unrestricted use, distribution, and reproduction in any medium, provided the original work is properly cited.

\section{References}

1. W. Barthlott, C. Neinhuis, Planta 202, 1 (1997).

2. C. Lee, Y. Nam, H. Lastakowski, J.I. Hur, S. Shin, A.-L. Biance, C. Pirat, C.-J. Kim, C. Ybert, Soft Matter 11, 4592 (2015).

3. B. Bhushan, Y.C. Jung, K. Koch, Langmuir 25, 3240 (2009).

4. L. Mishchenko, B. Hatton, V. Bahadur, J.A. Taylor, T. Krupenkin, J. Aizenberg, ACS Nano 4, 7699 (2010). 
5. A. Marmur, Biofouling 22, 107 (2006).

6. D. Quéré, Rep. Prog. Phys. 68, 2495 (2005).

7. A.K. Kota, G. Kwon, W. Choi, J.M. Mabry, A. Tuteja, Nat. Commun. 3, 1025 (2012).

8. T. Young, Philos. Trans. R. Soc. London 95, 65 (1805).

9. R.N. Wenzel, Ind. Eng. Chem. 28, 988 (1936).

10. J. Berthier, K.A. Brakke, The Physics of Microdroplets (John Wiley \& Sons, Inc. Hoboken, NJ, 2012).

11. A.B.D. Cassie, S. Baxter, Trans. Faraday Soc. 40, 546 (1944).

12. C. Dorrer, J. Rühe, Soft Matter 5, 51 (2009).

13. W. Choi, A. Tuteja, J.N. Mabry, R.E. Cohen, G.H. McKinley GH, J. Colloid Interface Sci. 339, 208 (2009).

14. C.W. Extrand, Langmuir 20, 5013 (2004).

15. P.S. Swain, R. Lipowsky, Langmuir 14, 6772 (1998).

16. G. McHale, Langmuir 23, 8200 (2007).
17. P. Papadopoulos, L. Mammen, X. Deng, D. Vollmer, H.-J. Butt, Proc. Natl. Acad. Sci. U.S.A. 110, 3254 (2013).

18. K.A. Brakke, Exp. Math. 1, 141 (1992).

19. K.A. Brakke, Surface Evolver Manual, Version 2.70 (2014) http://www.susqu.edu/brakke/evolver/html/ evolver.htm.

20. A. Sarkar, A.-M. Kietzig, Chem. Phys. Lett. 574, 106 (2013).

21. A.I. Hienola, P. M. Winkler, P.E. Wagner, H. Vehkamäki, A. Lauri, I. Napari, M. Kulmala, J. Chem. Phys. 126, 094705 (2007).

22. P.-G. de Gennes, F. Brochard-Wyart, D. Quere, Capillarity and Wetting Phenomena: Drops, Bubbles, Pearls, Waves (Springer Science+Business Media, New York, 2004).

23. H.P. Jennissen, Mat.-wiss. u.Werkstofftech. 42, 1111 (2011). 